\title{
Percutaneous Vertebral Augmentation for the Treatment of Symptomatic Schmorl's Nodes: Our Viewpoint and Experience
}

\section{TO THE EDITOR:}

It is with great interest that we read the article by He et al, "Percutaneous Vertebroplasty for Symptomatic Schmorl's Nodes: 11 Cases with Long-term Follow-up and a Literature Review", published in the 2017 February issue of Pain Physician (1).

Nowadays, percutaneous vertebral augmentation (PVA) is a valid therapeutic option in the management of severe back pain caused by osteoporotic and malignant vertebral compression fractures. PVA encompasses several techniques aimed at internal vertebral body stabilization with bone cement. Traditional percutaneous vertebroplasty (PVP) involves instillation of polymethylmethacrylate cement directly into the vertebral body, while percutaneous kyphoplasty (PKP) involves first creating a cavity using inflatable balloons to attempt more controlled cement delivery and improved cement interdigitation $(2,3)$. Despite the literature supporting the efficacy of PVA for treatment of vertebral fractures, few reports exist documenting its use in the treatment of symptomatic Schmorl's nodes (SNs) refractory to conservative treatment $(1,4,5)$.

This is a thoughtful and well-designed retrospective study which evaluated the safety and feasibility of PVP in the treatment of symptomatic SNs. The article suggests that magnetic resonance imaging (MRI) is the gold standard imaging technique to detect symptomatic SNs that vertebral body marrow changes around the nodes were T1 dark and T2 bright (1). The viewpoint of the author is right, but we have some concern about the MRI images presented in the article because the edematous rim around SNs is not significant in the vertebral body, especially in the fat-suppressed T2 weighted MRI image (Fig. 1). As we know, most SNs induce no symptoms. However, SNs are considered to be symptomatic if they present with an edematous rim around the nodes in the cancellous vertebral body with high signal intensity on a T2-weighted MRI and especially in the fat saturation sequence (6). Therefore, an MR examination is reserved for patients suspected of suffering from a symptomatic Schmorl's node on the basis of the absence of other major vertebral body abnormalities. Here, we present a typical case who underwent PKP for the treatment of symptomatic SNs. PKP was performed according to the procedure described by Yang et al (7) and biopsies around the nodes were collected for pathological examination. The MRI showed an impressive Schmorl node

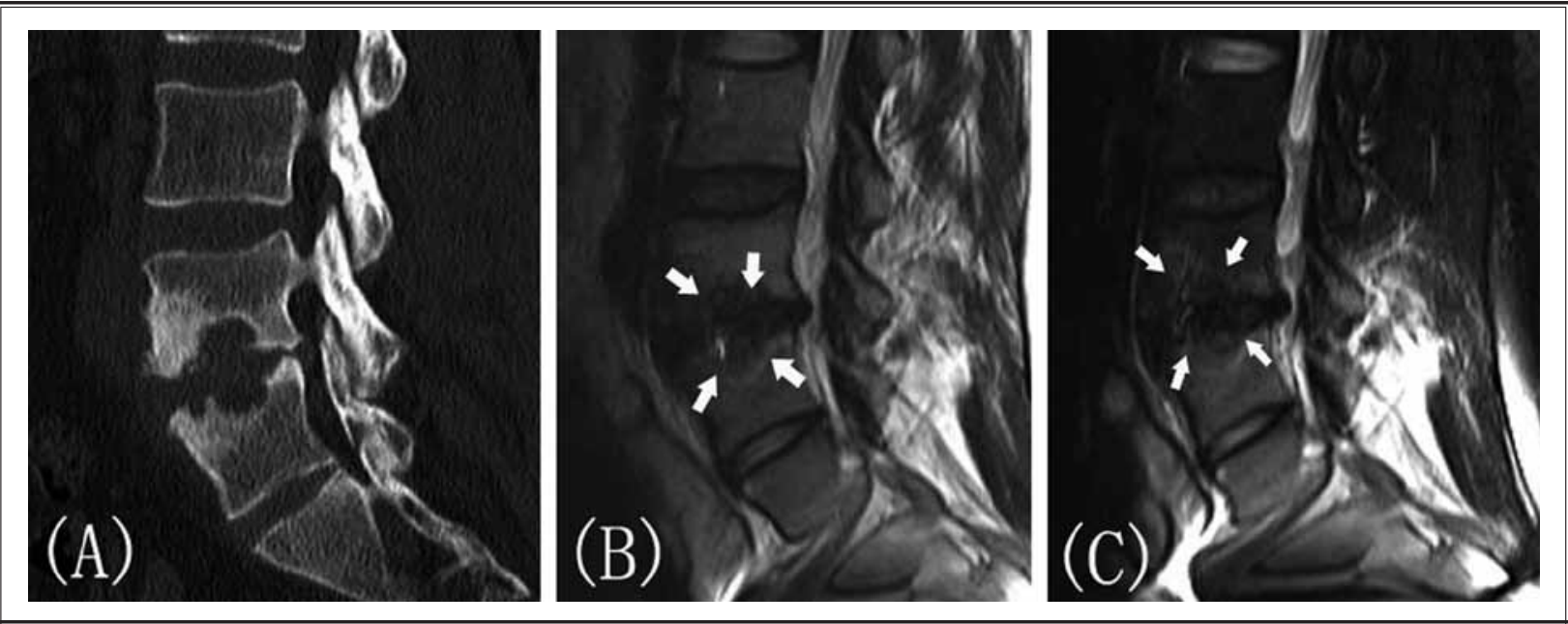

Fig. 1. Sagittal (A) CT images of the lumbar spine demonstrate a L4 inferior endplate Schmorl's node and a superior L5 endplate Schmorl's node. Sagittal T2 weighted (B) and fat suppressed T2 weighted MRI (C) demonstrate minor increased signal intensity in L4 and L5 vertebral bodies and edematous rim around the nodes is not significant, especially in the fat saturation sequence (White Arrows point at the insignificant edematous rim around the node). 

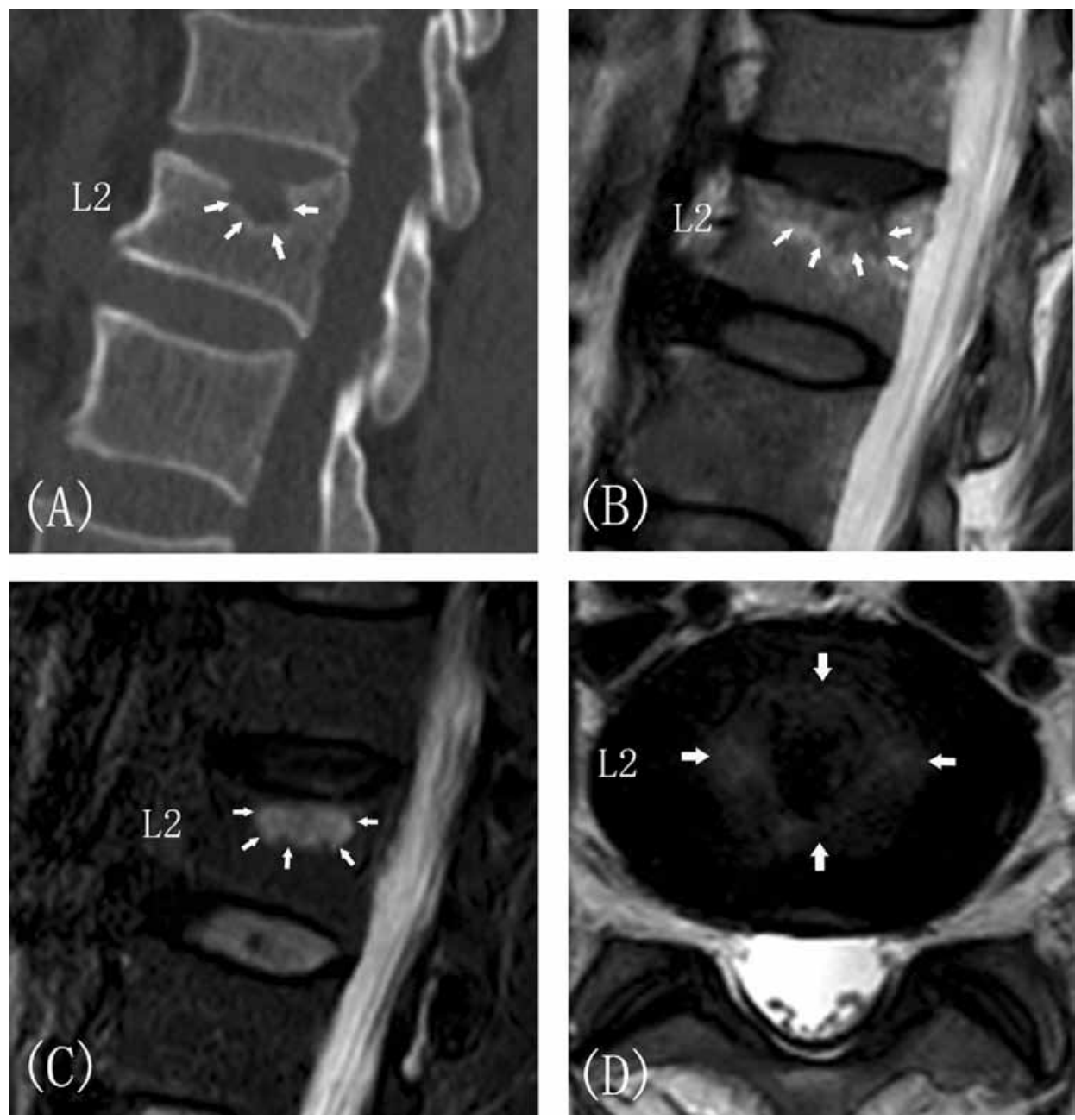

Fig. 2. A 54-year-old male patient was referred to the our orthopedic center with a 3-month history of incapacitating back pain; the symptoms did not benefit from conservative treatment such as analgesics, bed rest, and external bracing. Sagittal (A) computed tomography (CT) images of the lumbar spine demonstrate an L2 Schmorl's node (White Arrows). Sagittal T2 weighted (B) and fat-suppressed T2 weighted MRI (C) demonstrate an impressive Schmorl's node surrounded by a marked rim of high signal intensity in the L2 vertebral body (White arrows point at the edematous rim around the node). Axial T2-weighted MRI with fat suppression sequence $(D)$ in the L2 vertebra shows increased signal intensity in the vertebral body bone marrow surrounding the Schmorl's node (White arrows point at the edematous rim around the node).

surrounded by a marked rim of high signal intensity in the L2 vertebral body (Fig. 2) and it was confirmed by a histological examination that the MRI findings indicated the presence of inflammation and edema in the vertebral bone marrow (Fig. 3).

The results of this retrospective study show that PVP is an effective and safe procedure for the treatment of symptomatic SNs refractory to conservative therapy. Although thoughtful and well-prepared, the analgesic mechanism of PVP was not even not men- tioned in the article (1). We presume that symptomatic SNs represent a fresh intraosseous fracture in the vertebral body. Inflammatory change in the vertebral body marrow induced by intraosseous fracture and some biological reaction to the intraspongious disc materials might exist. Micromovements, inflammation and pressure on nociceptors within the edematous area probably induce back pain $(8,9)$. This suggests that symptomatic SNs could be treated with PVA, like osteoporotic vertebral fractures, with augmentation of the edema- 


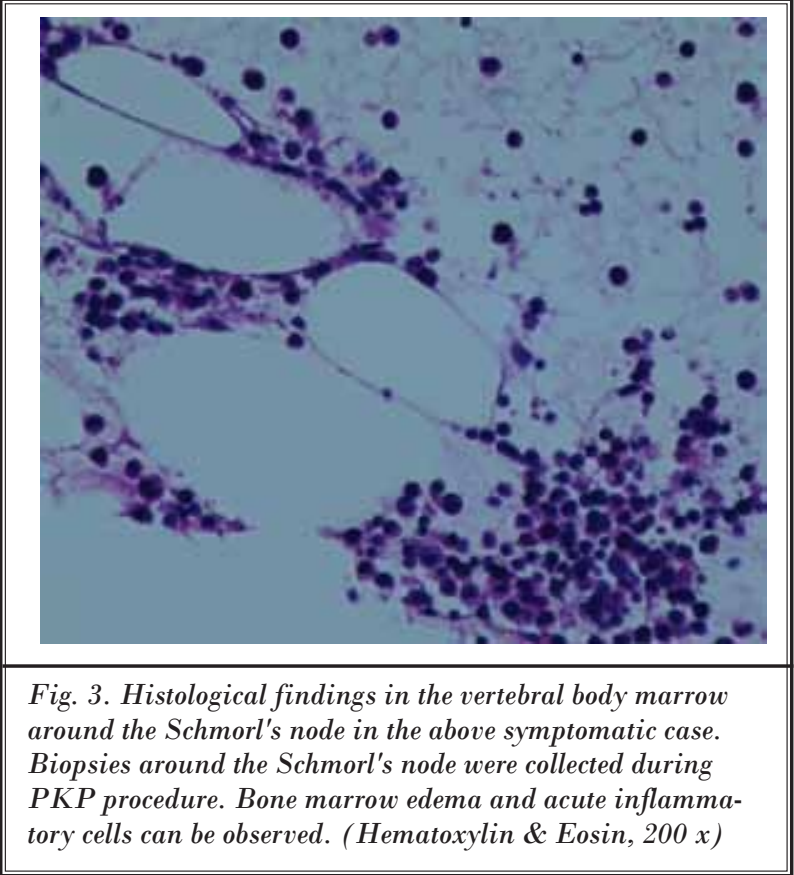

tous zone and not of the node itself $(9,10)$. The exact mechanism by which these PVA procedures achieve pain relief remains controversial. It is likely that pain relief is obtained through stabilization of the intraosseous fracture by the cement. Another explanation proposed is that the injected bone cement causes thermal necrosis and chemotoxicity to the intraosseous pain receptors $(11,12)$. We postulate that after the intraosseous fracture healing and subsidence of inflammation, the SNs may become asymptomatic, in analogy with old vertebral compression fractures.

It is known that the most important advantage of PKP over PVP is the ability to create a cavity into the vertebral body for the injection of a viscous cement with low pressure, significantly reducing the probability of cement leakage (7). As previous studies have shown that symptomatic SNs may represent an early stage of vertebral body fracture $(10,13)$, we propose the use of PKP over PVP as a therapeutic strategy in patients with incapacitating pain caused by symptomatic SNs which demonstrate significant signs of local edema and inflammation in MRI images. From January 2008 through December 2012, 32 patients suffering from symptomatic SNs underwent 43 PKP procedures in our orthopedic medical center. Outcome data including vertebral body height variation, Visual Analog Scales for pain, Oswes- try Disability Index and SF-36 questionnaire for function were collected preoperatively, postoperatively, and at 1-month, 6-months, 2-years, and 5-years after treatment. Significant improvements in all of the outcome measures were observed and maintained throughout the duration of follow-up. We found PKP is an effective and safe procedure in the treatment of symptomatic SNs refractory to conservative therapy.

In conclusion, symptomatic SNs should be treated similarly to vertebral compression fracture, and conservative treatments such as analgesics, bed rest, and external bracing should be the first choice (14). If conservative treatment fails, percutaneous minimally invasive vertebral augmentation procedures such as PVP and PKP might be indicated prior to anterior corpectomy and fusion $(9,10)$.

\section{Acknowledgements}

This project is supported by the National Natural Science Foundation of China (No.81301646 to Sun ZhiYong). The authors would like to thank the editors and reviewers for their review and constructive criticism in improving the manuscript.

Zhi-Yong Sun, MD

Department of Orthopaedic Surgery

The First Affiliated Hospital of Soochow University 188 Shizi St., Suzhou Jiangsu 215006, China

Xiao-Yu Zhu, MD

Department of Orthopaedic Surgery

The First Affiliated Hospital of Soochow University 188 Shizi St., Suzhou

Jiangsu 215006, China

Zhong-Lai Qian, MD

Department of Orthopaedic Surgery

The First Affiliated Hospital of Soochow University 188 Shizi St., Suzhou

Jiangsu 215006, China

Hui-Lin Yang, MD, PhD

Department of Orthopaedic Surgery

The First Affiliated Hospital of Soochow University 188 Shizi St., Suzhou

Jiangsu 215006, China

E-mail: soochowspine@139.com 


\section{References}

1. He SC, Zhong BY, Zhu HD, Fang W, Chen L, Guo JH, Deng G, Teng GJ. Percutaneous vertebroplasty for symptomatic Schmorl's Nodes: 11 Cases with long-term follow-up and a literature review. Pain Physician 2017; 20:69-76.

2. Zhang H, Xu C, Zhang T, Gao Z. Does percutaneous vertebroplasty or balloon kyphoplasty for osteoporotic vertebral compression fractures increase the incidence of new vertebral fractures? A Meta-analysis. Pain Physician 2017; 20:E13E28.

3. Zhan Y, Jiang J, Liao H, Tan H, Yang K. Risk factors for cement leakage after vertebroplasty or kyphoplasty: A meta-analysis of published evidence. World Neurosurg 2017. [Epub ahead of print]

4. Aparisi F. Vertebroplasty and kyphoplasty in vertebral osteoporotic fractures. Semin Musculoskelet Radiol 2016; 20:382-391.

5. Hasegawa K, Ogose A, Morita T, Hirata Y.
Painful Schmorl's node treated by lumbar interbody fusion. Spinal Cord 2004; 42:124-128.

6. Moustarhfir M, Bresson B, Koch P, Perozziello A, Barreau G, Schouman-Claeys E, Henry-Feugeas MC, Ou P, Dallaudière B. MR imaging of Schmorl's nodes: Imaging characteristics and epidemio-clinical relationships. Diagn Interv Imaging 2016; 97:411-417.

7. Yang $\mathrm{H}$, Liu $\mathrm{H}$, Wang $\mathrm{S}$, Wu K, Meng B, Liu T. Review of percutaneous kyphoplasty in China. Spine (Phila Pa 1976) 2016; 41: Suppl 19:B52-B8.

8. Mattei TA, Rehman AA. Schmorl's nodes: Current pathophysiological, diagnostic, and therapeutic paradigms. Neurosurg Rev 2014; 37:39-46.

9. Kyere KA, Than KD, Wang AC, Rahman SU, Valdivia-Valdivia JM, La Marca F, Park P. Schmorl's nodes. Eur Spine ] 2012; 21:2115-2121.
10. Fehlings MG. The safety of percutaneous vertebroplasty and kyphoplasty. J Neurosurg Spine 2009; 11:605-606.

11. Peng B, Wu W, Hou S, Shang W, Wang $X$, Yang Y. The pathogenesis of Schmorl's nodes. J Bone Joint Surg Br 2003; 85:879882.

12. Du J, Li X, Lin X. Kyphoplasty versus vertebroplasty in the treatment of painful osteoporotic vertebral compression fractures: Two-year follow-up in a prospective controlled study. Acta Orthop Belg . 2014; 80:477-486.

13. Boukhris R, Becker KL. Schmorl's nodes and osteoporosis. Clin Orthop Relat Res 1974; 104:275-280.

14. Burke KL. Schmorl's nodes in an American military population: Frequency, formation, and etiology. J Forensic Sci. 2012; 57:571-577. 